\title{
MEASURING THE DYNAMIC DISPLACEMENTS OF BRIDGES USING GEOPHONE DATA: APPLICATION AND VALIDATION ON A LIVELY FOOTBRIDGE
}

\author{
Paolo Borlenghi $^{1}$, George Piniotis ${ }^{2}$, Harris Perakis ${ }^{2}$, Vassilis Gikas ${ }^{2}$, Carmelo Gentile ${ }^{1}$ \\ ${ }^{1}$ Department of Architecture, Built environment and Construction engineering; Politecnico di Milano; \\ Piazza Leonardo da Vinci, 32 - 20133 Milan, Italy \\ e-mail: \{paolo.borlenghi, carmelo.gentile\}@polimi.it \\ ${ }^{2}$ School of Rural and Surveying Engineering; National Technical University of Athens; \\ 15780, Athens, Greece \\ e-mail: \{vgikas, hperakis\}@central.ntua.gr, piniotis@survey.ntua.gr
}

Keywords: Structural dynamic displacement, Cable-stayed bridge, Field test, Geophone, Signal integration

\begin{abstract}
Dynamic displacement is a highly valuable information for the evaluation of bridge safety and performance, providing data on the dynamic behaviour of the structure under service loads. However, its measure is often challenging and costly: traditional techniques require a fixed support and a direct connection (or a clear line-of-sight) with the target point that is rarely present in large-scale infrastructures, making their deployment in long-term monitoring often not feasible. In this study, an indirect measuring approach deploying an electro-dynamic velocity sensor (geophone) and digital filtering is adopted to measure human-induced dynamic displacements on a lively footbridge. These sensors have an excellent cost to performance ratio, do not require any powering and, overall, are easy to install and deploy for a long time. Furthermore, geophones measure velocities and consequently only "one integration step" is needed to obtain displacements. The field test was performed with the bridge partially open to pedestrian passages, the geophone was placed in different positions directly on the deck, and a reference measure of the vertical displacement was obtained from a laser-based system placed nearby. Appropriate digital filtering is then applied on the geophone data before the integration. The calibration of digital filters and the validation of the results was carried out comparing the reconstructed vertical displacements from geophone data and the directly measured vertical displacements from the state-of-art measuring device. Under the assumption of pure dynamic loading, some promising results were obtained, reaching a tenth of a millimetre accuracy for the integrated signals.
\end{abstract}




\section{INTRODUCTION}

Structural dynamic displacement is a highly valuable information for the evaluation of structural safety and performance, providing data on the dynamic behaviour of the structure under service loads. Furthermore, contemporary pedestrian bridges are often slender structures, prone to human-induced vibrations [1], making the control of displacement of great interest.

However, measuring dynamic displacement is often challenging and costly: traditional techniques require reference fixed points that are rarely found in large-scale structures, making the deployment in long-term monitoring often not feasible. Particularly, traditional displacement transducers, e.g. LVDT, requires direct contact both with the target measurement point and with external fixed support. Laser-based or radar-based systems, such as Laser Doppler vibrometer or Ground-based Microwave Interferometer [2], respectively, can provide noncontact measures of dynamic displacements but they still require a line-ofsight to the target point.

In recent years, some promising applications of low-cost satellite-based systems for structural displacement measures, such as GPS [3] or GNSS [4], were carried out but the lack of accuracy often remains an issue.

Another interesting approach is based on indirect measurements with accelerometers or velocimeters. These sensors are small, relatively low-cost, and easy to install and deploy for long time. The main drawback of these technologies is the need for signal processing when performing double or single integration for displacement reconstruction. Indeed, if direct integration is performed, the presence of noise in the recorded data and the inaccurate measures in the low-frequency range brings to erroneous estimation of displacements [5]. For this purpose, various digital filters can be applied: Butterworth filter [6], Baseline Correction [7], finite impulse response filter (FIR filter) [8], or Spectral Subtraction [9] are some examples.

Among the technologies that record accelerations and velocities, geophones are of interest for their cost to performance ratio and the null power demand. Geophones are a wellestablished technology in the field of seismology, mainly used for measuring ground motioninduced velocities, showing remarkable results in estimating the dynamic displacements [5]. In addition, geophones are well-suited sensors for Operation Modal Analysis of civil engineering structures (see e.g. [10]), making their deployment in a monitoring system of twofold interest: (a) identifying the modal characteristics of the structure; (b) monitoring the maximum displacements induced by operational loads.

More recently, geophones were widely deployed to evaluate the deformations that occur to railway track foundations, during the passage of trains [11-12]. Bowness et al. [6] describe a technique to filter and integrate geophone signals in the context of railway track monitoring. Firstly, the signals are deconvoluted to consider the frequency-dependent sensitivity and the phase lag of the sensor. Indeed, these instruments have a limited linear frequency range, above its natural frequency, typically between 4 and $12 \mathrm{~Hz}$, and when dealing with excitations that have a dominant frequency close to the natural frequency of the sensor, deconvolution is always needed. Secondly, a band-pass filter with a range of $0.3-15 \mathrm{~Hz}$ is applied: the highpass removes the low-frequency drift while the low-pass removes noise at high frequencies. However, defining the cut-off frequency can be challenging, considering that the filter becomes unstable when approaching $0 \mathrm{~Hz}$. Bowness et al. [6] define the lowest cut-off frequency equal to $0.2 \mathrm{~Hz}$.

Notwithstanding, the promising applications of geophones in the monitoring of railway track deformations or recording of strong motion-induced displacements, very few studies are 
present in the literature for the application of geophones to record structural dynamic displacements of bridges.

The present paper investigates the application of geophones in the measurement of dynamic displacement of pedestrian bridges. The procedure was exemplified on a cablestayed pedestrian bridge: the human-induced vibrations are recorded in the same point with a geophone and a state-of-art technology for measuring dynamic displacement (i.e. a Laser Tracker). Therefore, to estimate the dynamic displacement from the geophone data, digital filtering was applied before signal integration. The validation of the results and the calibration of the digital filter was performed through the comparison between reconstructed (from geophone data) and directly measured (from the Laser Tracker) displacements. Particularly, the following steps were involved:

(i) performing a field test on the selected cable-stayed bridge, measuring the humaninduced vibrations with multiple setups (subsequent measures in different points) deploying a geophone and a state-of-art measuring device;

(ii) performing the deconvolution of the geophone data with SEISMOWIN [13] software;

(iii) selecting and calibrating a digital filtering method (i.e. pass-band filter [6]) to minimise the difference between the measured and reconstructed dynamic displacements; and

(iv) consequently, developing a MATLAB-based algorithm to perform the filtering and the integration of geophone data for long-term monitoring purposes.

\section{ADOPTED MEASURING DEVICES}

Geophones are electro-dynamic velocity transducers that produce an output signal in voltage proportional to the recorded speed. The signal is generated by the relative movements of a coil suspended around a core magnet. Therefore, geophones are passive sensors and do not require any power supply. The main drawbacks of this technology are the limited frequency range and the limited excursion of the coil. In the low frequency range - below the resonant frequency of the sensors - the signal is attenuated, and a proper signal deconvolution must be performed. The limited excursion is given by the limited length of the suspended system.

(a)

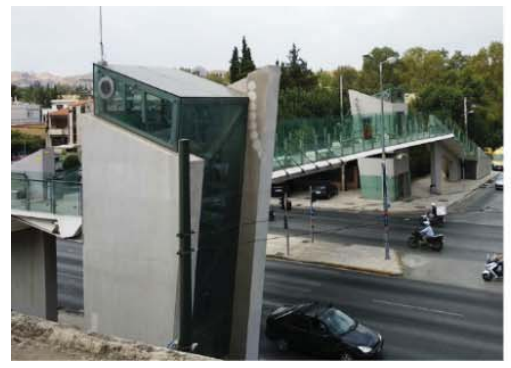

(b)

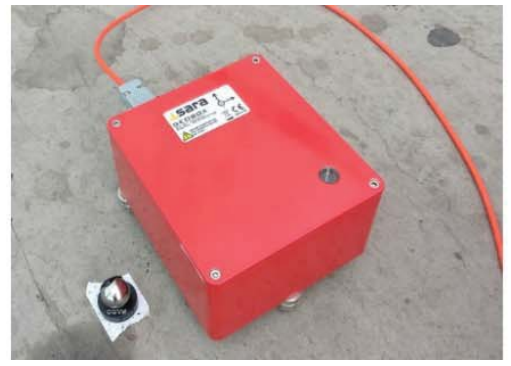

(c)

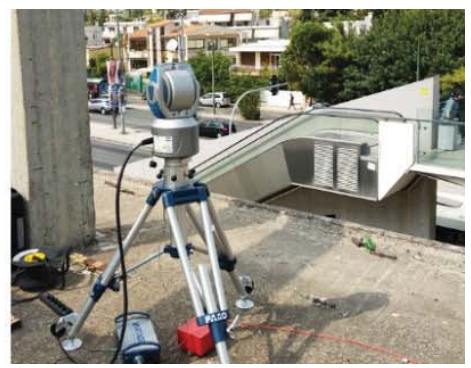

(d)

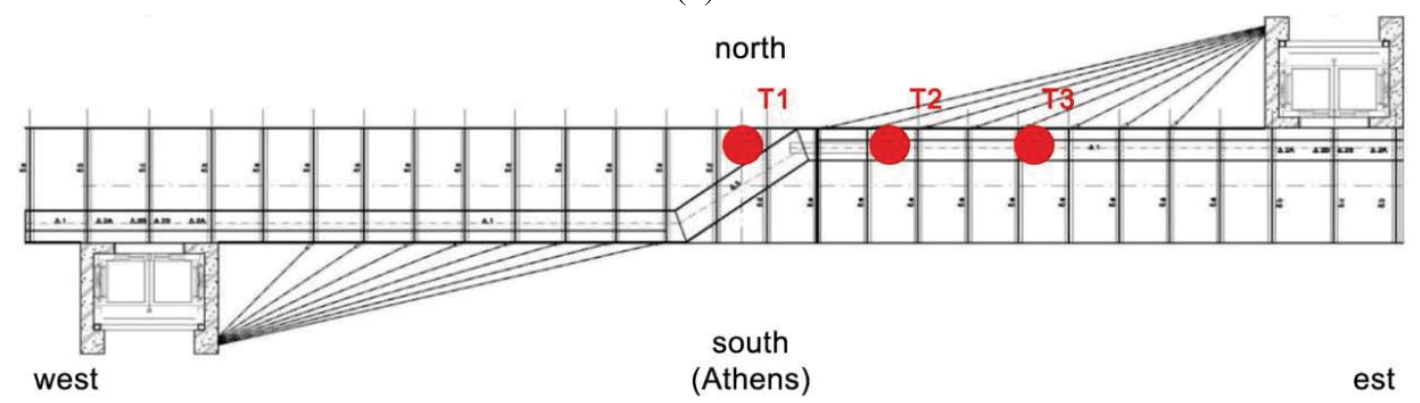

Figure 1: (a) Tested bridge; (b) Laser Tracker target and Geophone; (c) Laser Tracker; (d) Experimental setup. 
The indirect measures of displacement were conducted with a high-sensitive seismometer (Fig. 1b), namely a SARA GEOBOX SS45 (nominal sensitivity $78 \mathrm{~V} / \mathrm{ms}^{-1}$; natural frequency $4.5 \mathrm{~Hz}$ ). These specific geophones are of interest for the very good performance in the lowfrequency range with a usable dynamic band - before an appropriate signal deconvolution defined by the manufacturer of $0.2-400 \mathrm{~Hz}$.

The direct measurements of displacements were conducted with a laser tracker, namely the FARO Vantage (Fig. 1c). The Vantage is a portable, three dimensional coordinate measuring instrument that determines the position of a special Spherically Mounted Retroreflector (SMR), through the extremely accurate measurement of a zenith angle, an azimuth angle and a radial distance from the instrument. It uses a phase shift Absolute Distance Meter (ADM), providing a Maximum Permissible Error (MPE) accuracy of $\pm 16 \mu \mathrm{m}+0.8 \mu \mathrm{m} / \mathrm{m}$ for the radial distance measurements, while its angular Maximum Permissible Error (MPE) accuracy is $\pm 20 \mu \mathrm{m}+5 \mu \mathrm{m} / \mathrm{m}$. According to FARO, the typical performance of the sensor is half the MPE values. Vantage has a working range of $0 \mathrm{~m}$ to $80 \mathrm{~m}$ and it is capable of making static and dynamic measurements as well, since its maximum sampling frequency is $1 \mathrm{kHz}$.

\section{BRIDGE DESCRIPTION AND TEST PROGRAMME}

The test bridge (Fig. 1a), named "Bridge of dreams", is a cable stayed, composite bridge located in the North of Athens, Greece, crossing over the main road that leads to the Olympic Stadium Complex [14]. The bridge is suspended from two composite pylons using 16 fullylocked galvanized cables, anti-symmetrically distributed on each side of the structure. It has an overall length of $76.0 \mathrm{~m}$, a free span of $31.5 \mathrm{~m}$ and is $4.0 \mathrm{~m}$ wide.

(a) T1: Disp. LT

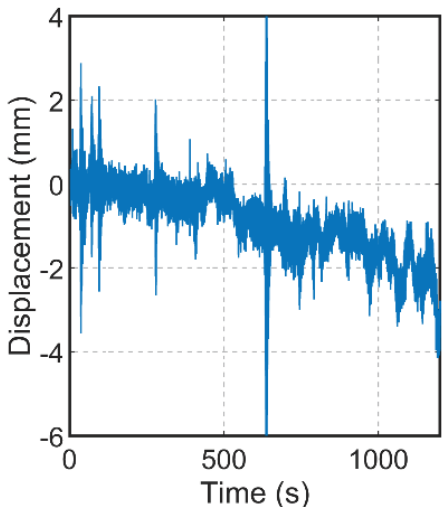

(d) T1: Disp. LT $\left(f_{\mathrm{c}}=0.2 \mathrm{~Hz}\right)$

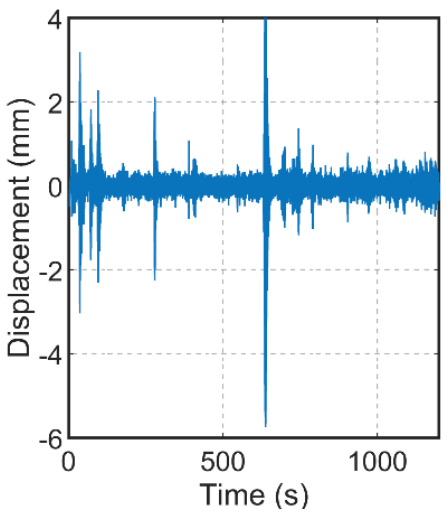

(b) T2: Disp. LT

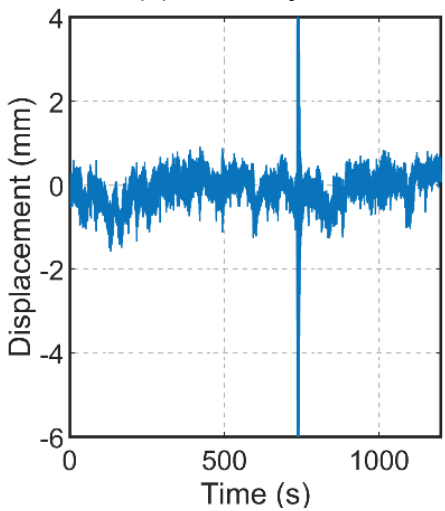

(e) T2: Disp. LT $\left(f_{\mathrm{c}}=0.2 \mathrm{~Hz}\right)$

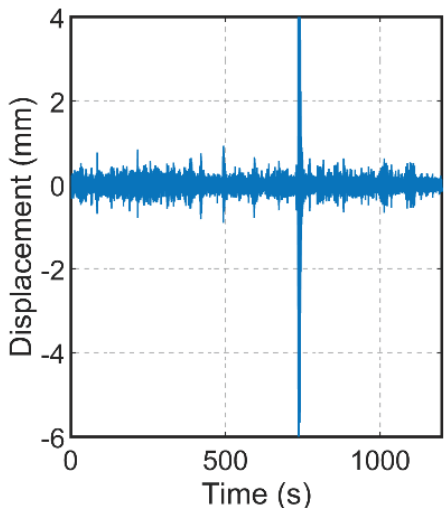

(c) T3: Disp. LT

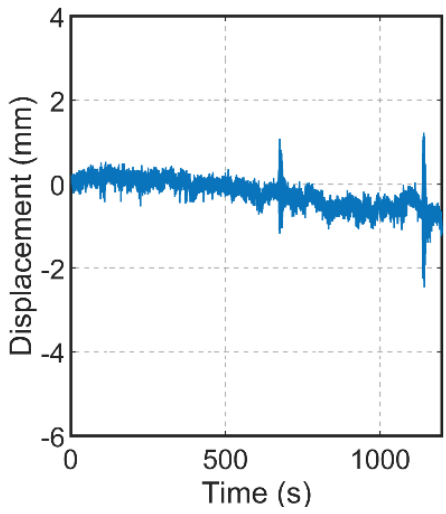

(f) T3: Disp. LT $\left(f_{\mathrm{c}}=0.2 \mathrm{~Hz}\right)$

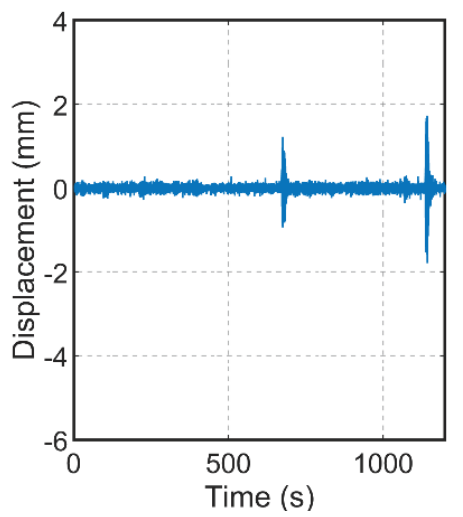

Figure 2: Laser tracker time-series of the 3 measured points: comparison between the non-filtered data (a-c) and the filtered data with a Bandpass Butterworth Filter with a lower cut-off frequency $\left(f_{\mathrm{c}}\right)$ of $0.2 \mathrm{~Hz}(\mathrm{~d}-\mathrm{f})$. 
The field test was performed with the bridge partially open to pedestrian passages; the geophone was placed in different positions directly on the deck (Fig. 1d), and a reference measure of the vertical displacement was obtained from the laser tracker placed on the roof of a nearby building (Fig. 1c). Particularly the test involved the following steps: (i) positioning of the state-of-art measuring device on the top of a nearby building to obtain a clear line-ofsight to the target point (the small sphere in Fig. 1b); (ii) positioning of the geophone (the red box in Fig. 1b) and the target point in the selected position (Fig. 1d); (iii) recording of humaninduced vibration with both devices for 1200 seconds with a sampling rate of $200 \mathrm{~Hz}$.

\section{SIGNAL PROCESSING AND DATA ANALYSIS}

The calibration of digital filters and the validation of the results was carried out comparing the reconstructed vertical displacements from geophone data and the directly measured vertical displacements from the laser tracker (LT).

The recorded time-series of displacement from the LT are shown in Fig. 2. Figs. 2a-c illustrates the raw displacement data obtained just subtracting the initial recorded value. As mentioned earlier, the test was performed with the bridge partially open to pedestrian passages. During the test the number of people that were walking or staying (several benches are present on the bridge deck) changed continuously, creating an overall increase of quasistatic loads; this effect is particularly visible in the first recording (T1, Fig. 2a). To obtain a pure dynamic displacement, the effects of the quasi-static loads and the noise in the high frequency range were removed with a pass-band filter with cut-off frequencies of $0.2 \mathrm{~Hz}$ and $15 \mathrm{~Hz}$ (Figs. 2d-f).

(a) T1: Disp. LT

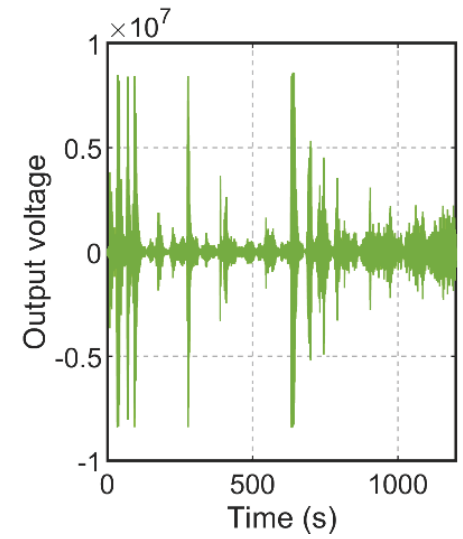

(d) T1: Disp. LT $\left(f_{\mathrm{c}}=0.2 \mathrm{~Hz}\right)$

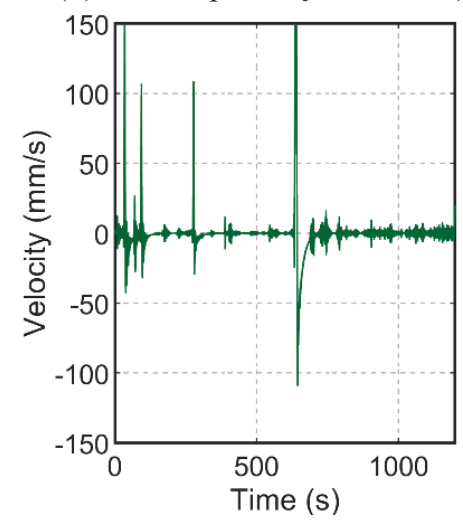

(b) T2: Disp. LT

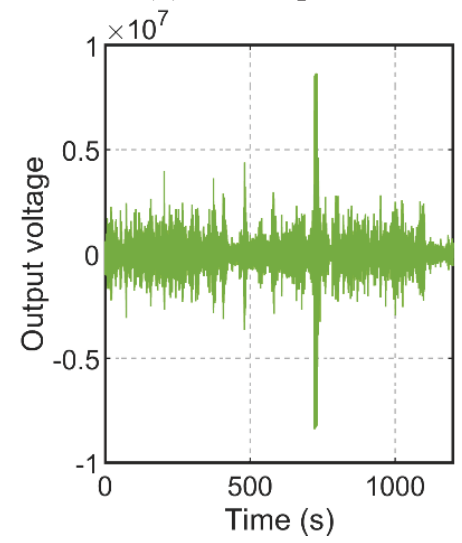

(e) T2: Disp. LT $\left(f_{\mathrm{c}}=0.2 \mathrm{~Hz}\right)$

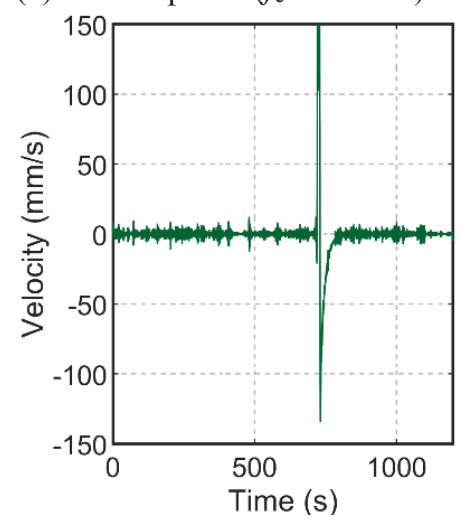

(c) T3: Disp. LT

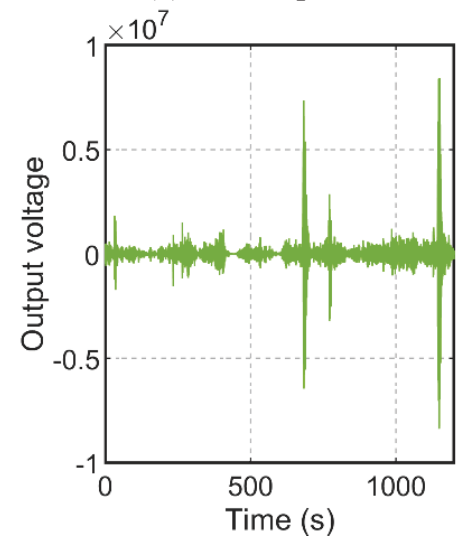

(f) T3: Disp. $\operatorname{LT}\left(f_{\mathrm{c}}=0.2 \mathrm{~Hz}\right)$

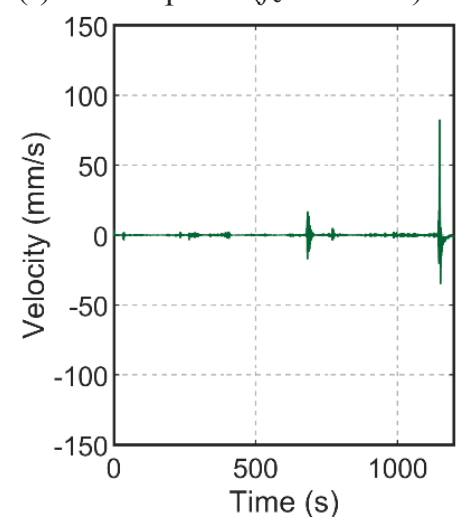

Figure 3: Geophone time-series from of the 3 measured points: comparison between the raw data (a-c) and the deconvoluted velocity data (d-f). 
The recorded signals from the geophone are shown in Fig 3. Figs. 3a-c illustrates the raw output voltages: the sharp peaks, particularly visible in the first recording, are caused by high dynamic loads given by people running on the deck. In these instances, the signal reached the threshold given by limited excursion of the coil, resulting in a clipping of the recorded data. This is particularly visible in the deconvoluted velocity data (Figs. 3d-f): the clipped signals results in abnormal speed increases that must be removed or ignored.

(a) Vel. GEO $\left(f_{\mathrm{c}}=0.2 \mathrm{~Hz}\right)$

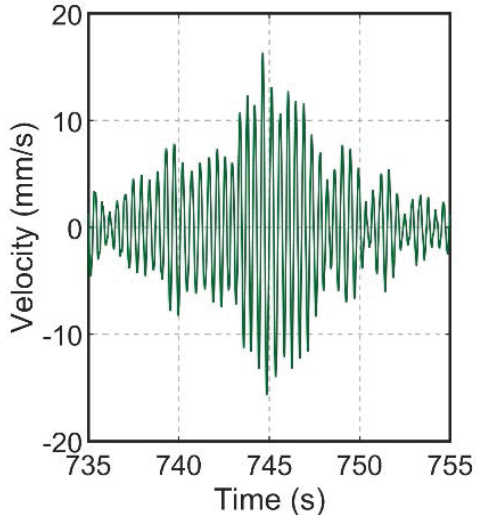

(d) Vel. GEO $\left(f_{\mathrm{c}}=0.6 \mathrm{~Hz}\right)$

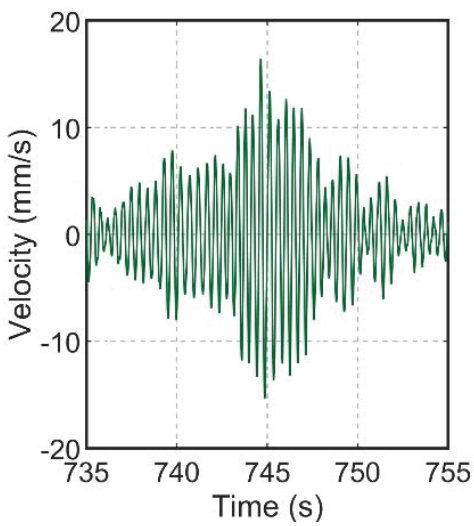

(b) Disp. GEO $\left(f_{\mathrm{c}}=0.2 \mathrm{~Hz}\right)$

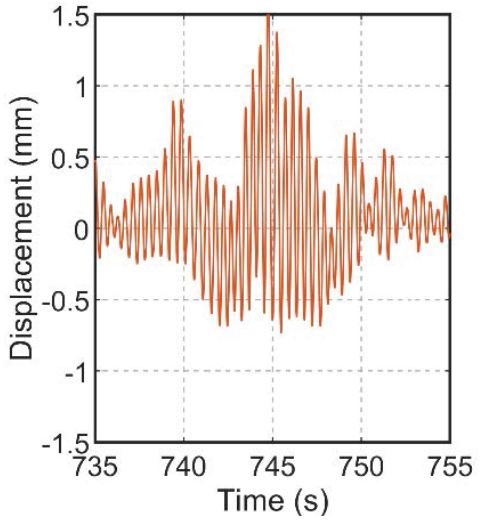

(e) Disp. GEO $\left(f_{\mathrm{c}}=0.6 \mathrm{~Hz}\right)$

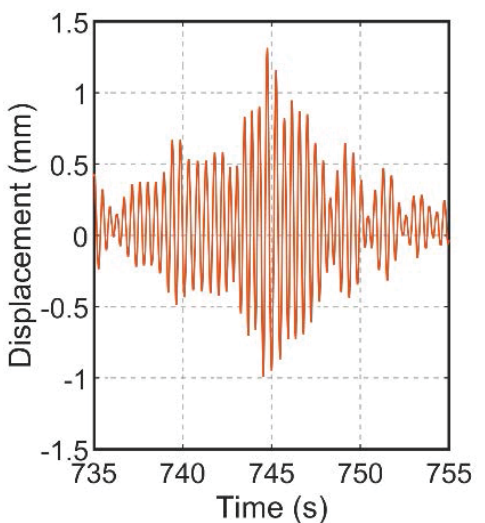

(c) Disp. LT $\left(f_{\mathrm{c}}=0.2 \mathrm{~Hz}\right)$

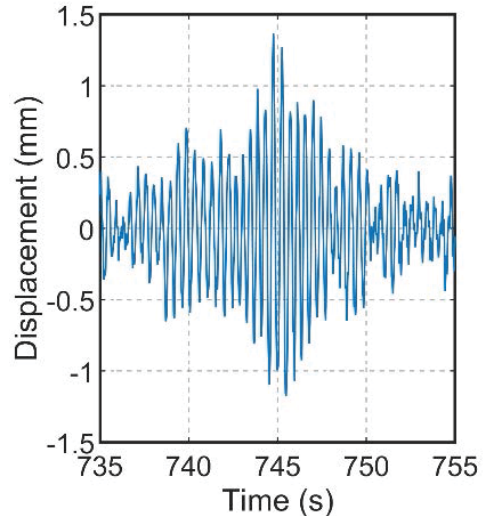

(f) Disp. LT $\left(f_{\mathrm{c}}=0.2 \mathrm{~Hz}\right)$

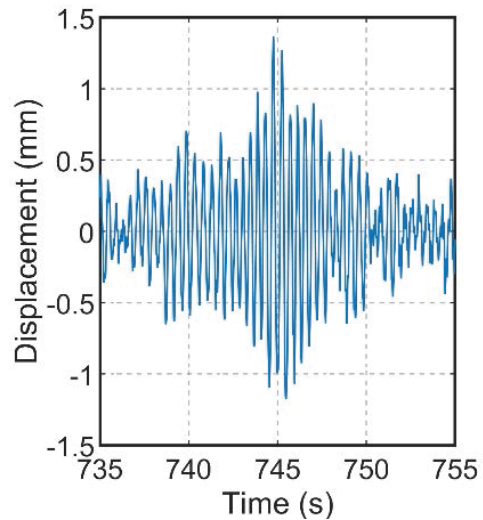

Figure 4: Time-series of $20 \mathrm{sec}$ from T1: geophone velocities (a,d) and reconstructed displacements (b, e) with different cut-off frequency $\left(f_{c}\right)$ and reference laser tracker displacements $(c, f)$.

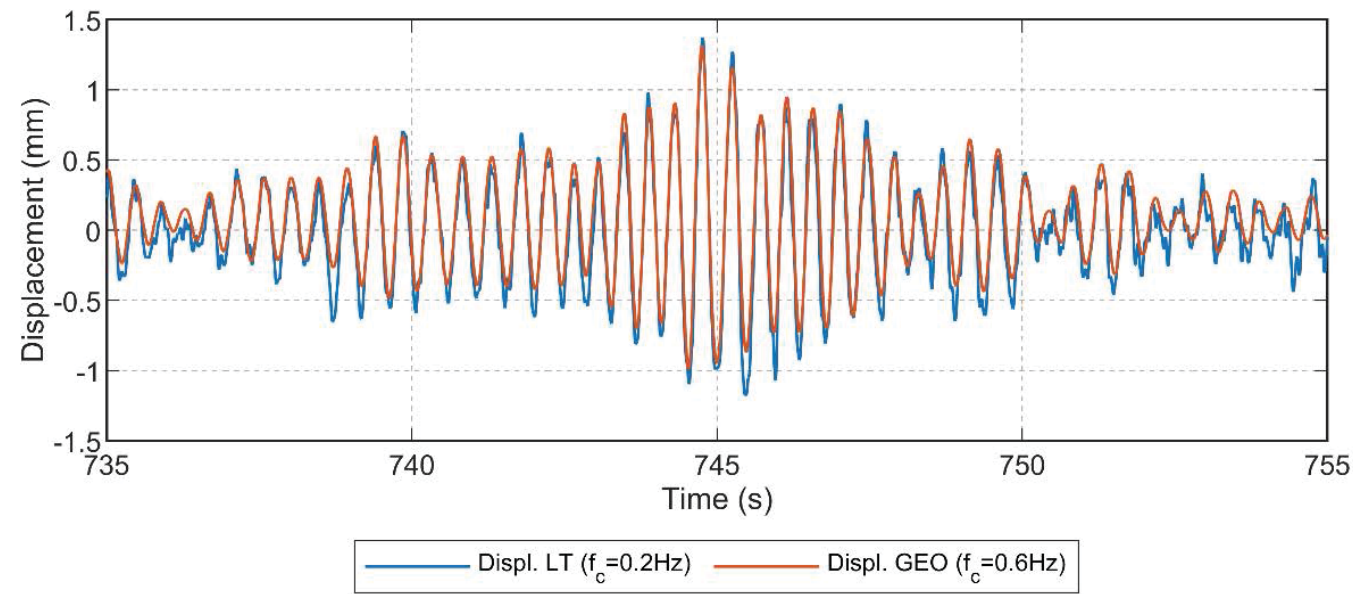

Figure 5: Time-series of $20 \mathrm{sec}$ from T1: comparison between the reconstructed displacements from the geophone $\left(f_{\mathrm{c}}=0.6 \mathrm{~Hz}\right)$ and the reference laser tracker displacements $\left(f_{\mathrm{c}}=0.2 \mathrm{~Hz}\right)$. 
Subsequently, a pass-band Butterworth filter was applied on the velocity data before the integration. The difference between the references and the reconstructed displacements were minimised with a manual tuning of the cut-off frequencies of the filter. To exemplify the calibration procedure, in Fig. 4 and Fig. 6 are shown few seconds of the time-series recorded at T1 and T3. Firstly, a lower cut-off frequency $\left(f_{\mathrm{c}}\right)$ of $0.2 \mathrm{~Hz}$ was adopted showing some distortion in the reconstructed displacements (Figs. 4b, 6b). The best match was obtained with $f_{\mathrm{c}}=0.6 \mathrm{~Hz}$.

(a) Vel. GEO $\left(f_{\mathrm{c}}=0.2 \mathrm{~Hz}\right)$

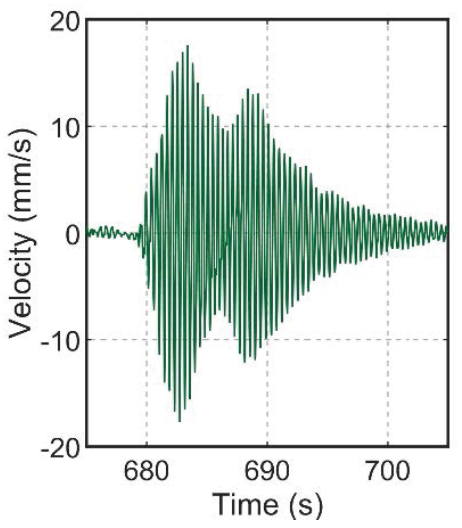

(d) Vel. GEO $\left(f_{\mathrm{c}}=0.6 \mathrm{~Hz}\right)$

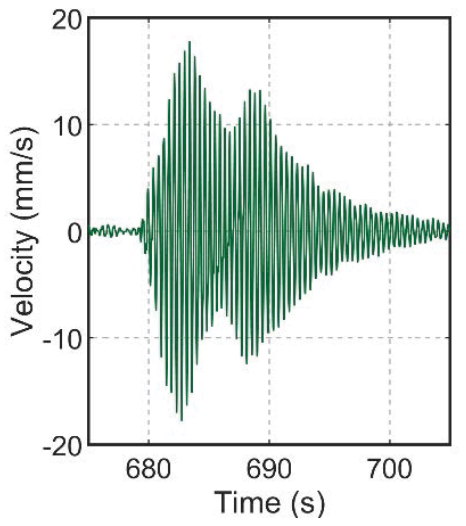

(b) Disp. GEO $\left(f_{\mathrm{c}}=0.2 \mathrm{~Hz}\right)$

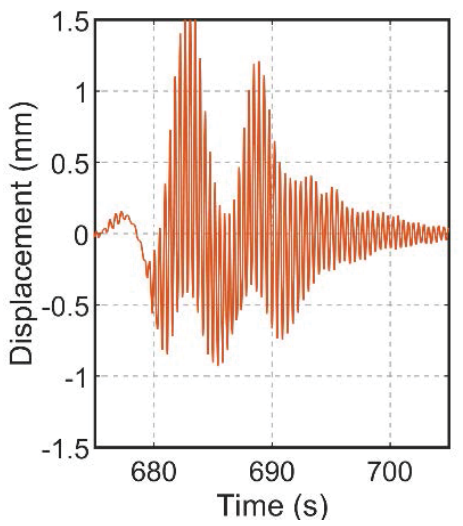

(e) Disp. GEO $\left(f_{\mathrm{c}}=0.6 \mathrm{~Hz}\right)$

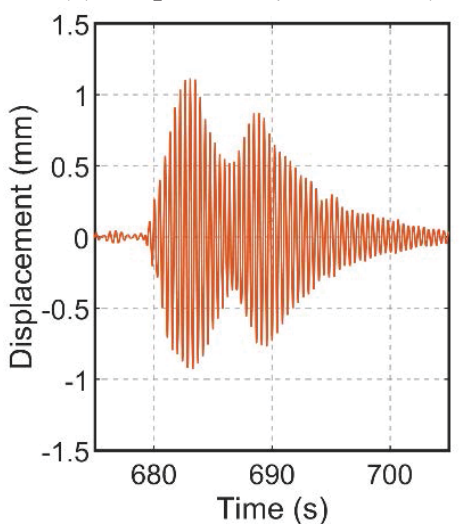

(c) Disp. LT $\left(f_{\mathrm{c}}=0.2 \mathrm{~Hz}\right)$

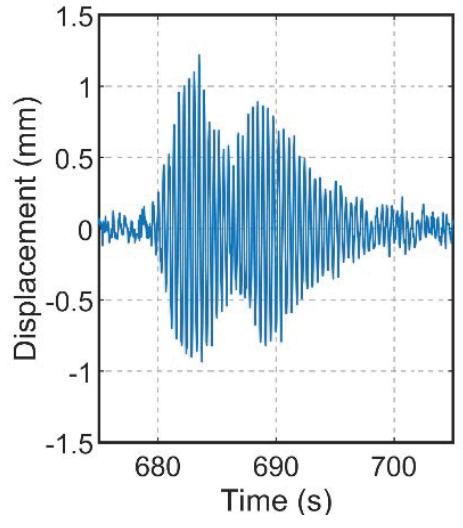

(f) Disp. LT $\left(f_{\mathrm{c}}=0.2 \mathrm{~Hz}\right)$

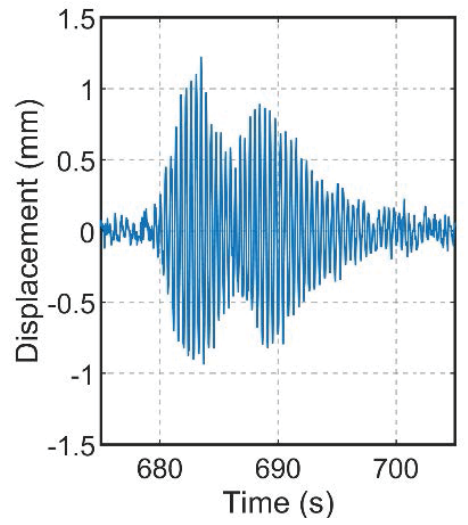

Figure 6: Time-series of $30 \mathrm{sec}$ from T3: geophone velocities (a, d) and reconstructed displacements (b, e) with different cut-off frequency $\left(f_{c}\right)$ and reference laser tracker displacements $(c, f)$.

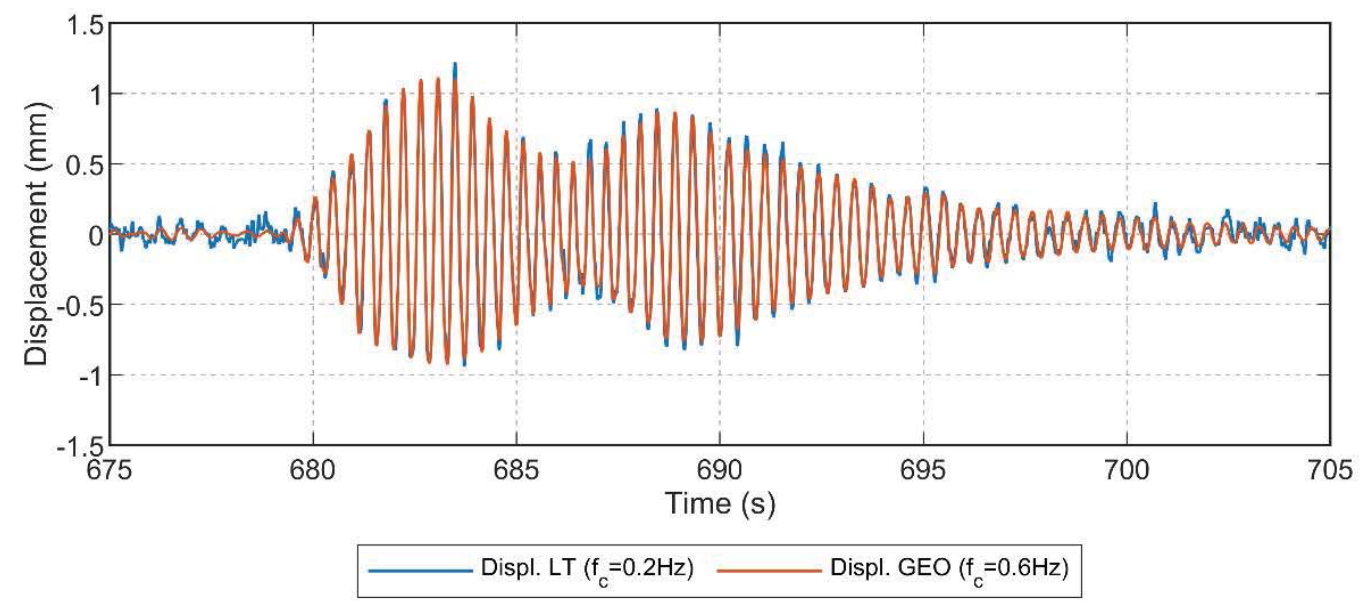

Figure 7: Time-series of $30 \mathrm{sec}$ from T3: comparison between the reconstructed displacements from the geophone $\left(f_{\mathrm{c}}=0.6 \mathrm{~Hz}\right)$ and the reference laser tracker displacement $\left(f_{\mathrm{c}}=0.2 \mathrm{~Hz}\right)$. 


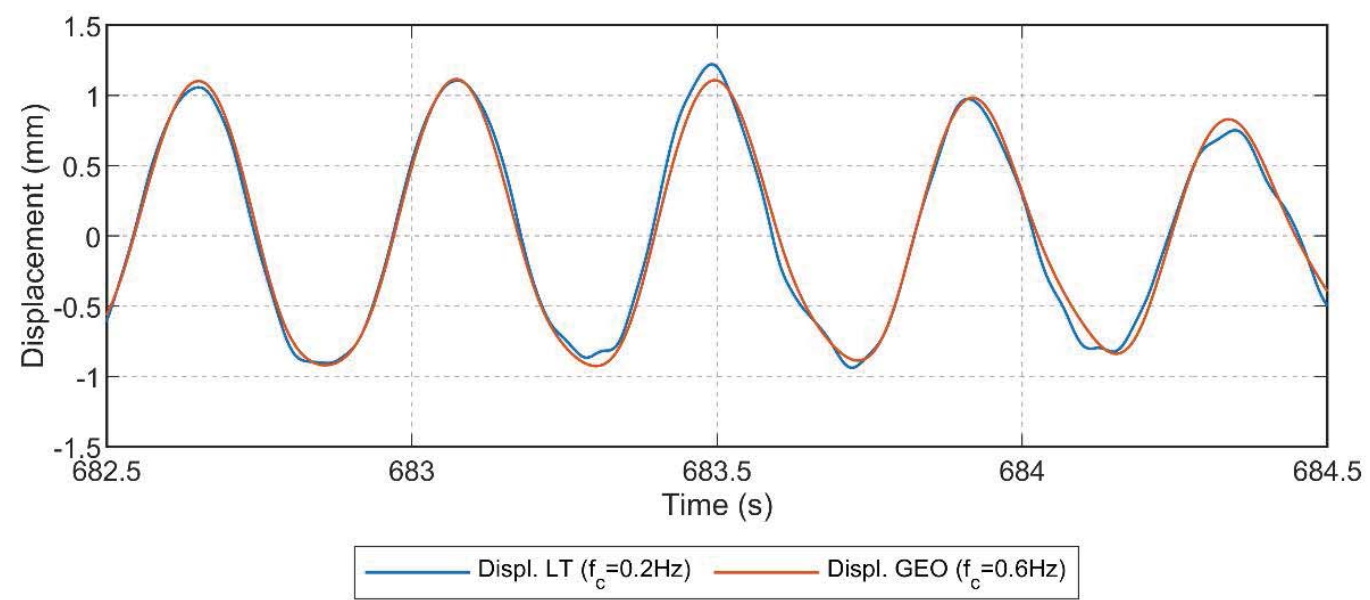

Figure 8: Focus on a time-series of $2 \mathrm{sec}$ from T3: comparison between the reconstructed displacements from the geophone $\left(f_{\mathrm{c}}=0.6 \mathrm{~Hz}\right)$ and the reference laser tracker displacement $\left(f_{\mathrm{c}}=0.2 \mathrm{~Hz}\right)$.

Figs. 5 and 7 demonstrates the very good agreement between the reconstructed and the reference displacements.

\section{CONCLUSIONS}

Previous studies have demonstrated the successful application of digital signal processing on the velocity data recorded with geophones to reconstruct the displacements of railway track foundations and strong-motion induced displacements. In this study, an application of digital filtering for the reconstruction of human-induced displacements on a pedestrian cable stayed bridge is illustrated. The results from the investigated structure suggest the following conclusions:

1) Under the assumption of pure dynamic loading, it is possible to reconstruct the induced displacements from the geophone data by simply applying a Butterworth filter before the integration;

2) Approximately, a tenth of a millimetre accuracy was obtained for the integrated signals (Fig. 8);

3) The cut-off frequencies of the filter are connected to the dynamic characteristics of the investigated structure and to its operational loads;

4) Consequently, from a Structural Health Monitoring perspective, the filter calibration must be performed each time a long-term monitoring is installed;

5) Despite the generally very good accuracy in terms of reconstructed displacement, the sensor reached its measurement threshold several times, making impossible to reconstruct the maximum displacements experienced during the test. Further investigations are needed to reconstruct the clipped signals.

The promising results suggest the future application of the proposed signal processing strategy for the continuous monitoring of the dynamic displacement of bridges. In addition, more tests on structures with higher and lower stiffness have already been planned.

\section{REFERENCES}

[1] E. Caetano, Á. Cunha, F. Magalhães, C. Moutinho, Studies for controlling humaninduced vibration of the Pedro e Inês footbridge, Portugal. Part 1: Assessment of dynamic behaviour, Engineering Structures 32(4), 1069-1081, 2010. 
[2] C. Gentile, G. Bernardini, An interferometric radar for non-contact measurement of deflections on civil engineering structures: Laboratory and full-scale tests, Structure and Infrastructure Engineering 6(5), 521-534, 2010.

[3] F. Moschas, S. Stiros, Measurement of the dynamic displacements and of the modal frequencies of a short-span pedestrian bridge using GPS and an accelerometer, Engineering Structures 33(1), 10-17, 2011.

[4] J. Yu, X. Meng, X. Shao, B. Yan, L. Yang, Identification of dynamic displacements and modal frequencies of a medium-span suspension bridge using multimode GNSS processing, Engineering Structures 81, 432-443, 2014.

[5] D.M. Boore, J.J. Bommer, Processing of strong-motion accelerograms: needs, options and consequences, Soil Dynamics and Earthquake Engineering 25, 93-115, 2005.

[6] D. Bowness, A. C. Lock, W. Powrie, J. A. Priest, D. J. Richards, Monitoring the dynamic displacements of railway track, in: Proceedings of the Institution of Mechanical Engineers, Part F: Journal of Rail and Rapid Transit 221(1), 13-22, 2007.

[7] D. M. Boore, Effect of baseline corrections on displacements and response spectra for several recordings of the 1999 Chi-Chi, Taiwan, earthquake, Bulletin of the Seismological Society of America 91(5), 1199-1211, 2001.

[8] H. S. Lee, Y. H. Hong, H. W. Park, Design of an FIR filter for the displacement reconstruction using measured acceleration in low-frequency dominant structures, International Journal for Numerical Methods in Engineering 82(4), 403-434, 2010.

[9] B. Coelho, P. Hölscher, F. Barends, Enhancement of double integration procedure through spectral subtraction, Soil Dynamics and Earthquake Engineering 31(4), 716$722,2011$.

[10] C. Gentile, A. Ruccolo, F. Canali, Continuous monitoring of the Milan Cathedral: dynamic characteristics and vibration-based SHM, Journal of Civil Structural Health Monitoring 9(5), 671-688, 2019.

[11] J. A. Priest, W. Powrie, L. Yang, P. J. Grabe, C. R. I. Clayton, Measurements of transient ground movements below a ballasted railway line, Geotechnique 60(9), 667$677,2010$.

[12] B. Coelho, P. Hölscher, J. Priest, W. Powrie, F. Barends, An assessment of transition zone performance, in: Proceedings of the Institution of Mechanical Engineers, Part F: Journal of Rail and Rapid Transit 225(2), 129-139, 2011.

[13] SARA electronic instruments, SEISMOWIN front-end 1.4.0, Perugia, Italy.

[14] V. Gikas, P. Karydakis, T. Mpimis, G. Piniotis, H. Perakis, Structural integrity verification of cable stayed footbridge based on FEM analyses and geodetic surveying techniques, Survey Review 48(346), 1-10, 2016. 